\title{
Immunogenetic Control of the Response of Female Mice to Male Tissue Grafts
}

\author{
Jane S. Schultz ${ }^{1,2}$, Roberta DeMott-Friberg ${ }^{1}$, and Theodore F. Beals ${ }^{1,2}$ \\ ${ }^{1}$ Veterans Administration Medical Center, Ann Arbor, Michigan 48105 \\ ${ }^{2}$ Departments of Human Genetics and Pathology, University of Michigan, Ann Arbor, Michigan
}

Inbred strains of mice which carry recombinant $H-2$ genomes have been utilized to locate the site of $H-2$ control of rejection of male liver tissue grafts by females of the same strain. When the $K$ end of the $H-2$ complex carries the $H-2^{b}$ allele, the strongest immunological response is evoked in female animals. However, there is a clear participation of the $D$ end of the $H-2$ complex in immune response to these grafts. The background genome also plays a significant role in graft rejection in some strains. The data presented lead to the hypothesis that control over rejection of these grafts is a complex interaction of multiple genetic factors.

The immunological phenomenon of rejection of male skin grafts by female mice of the same inbred strain is well documented (Gasser and Silvers 1972). This reaction is believed to result from immunological reactivity against the H-Y antigen of the male. The $H-2$ complex, or a gene closely linked to that complex, exerts a major control on the female's ability to reject male skin (Gasser and Silvers 1971, Bailey and Hoste 1971) and capacity to generate cytotoxic killer T lymphocytes against the $Y$ antigen of male cells (von Boehmer et al. 1978). For example, animals carrying the $H-2^{b}$ haplotype acutely reject male grafts while animals carrying the $H-2^{a}$ haplotype do not. $\mathrm{H}-2^{b}$ animals are also strong generators of anti-H-Y cytotoxic T lymphocytes. However, attempts to locate the female-vs-male skin-graft response to a specific region of the $H-2$ complex have produced conflicting results (Bailey 1971). Female B10.A (2R) and B10.A (4R) mice, which are recombinants of the $K$ end of $H-2^{a}$ and the $D$ end of $H-2^{b}$, react in the same manner as $H-2^{a}$ mice while recombinants between the $K$ end of $H-2^{b}$ and the $D$ end of $H-2^{a}$ react in a comparable manner to $H-2^{b}$ females in skin-graft rejection. These data suggest that the response to $\mathrm{H}-\mathrm{Y}$ is located at or closely linked to the $\mathrm{K}$ end of the $\mathrm{H}-2$ complex (Bailey 1971, Stimpfling and Reichert 1971). However, females of the HTG strain give an $H-2^{b}$-like skin-graft response even though they carry the $K$ region of the $H$ $2^{d}$ haplotype and the $D$ region of the $H-2^{b}$ haplotype. It may be concluded from these data that more than one locus is controlling the immune response of the female mice to grafts of male skin. In fact, female mice of the same $H$-2 type sometimes show significantly different degrees of response to male skin grafts. 
The immunogenicity of the H-Y antigen also appears to be influenced by the $\mathrm{H}-2$ type of the skin-graft donor (Wachtel et al. 1973). When $(B 10 \times B 10 . B R) F_{1}$ females are challenged with skin grafts from $\mathrm{B} 10$ males, these grafts are rejected at a different rate than B10.BR male skin grafts. Since the only difference in these strains is in the $H-2$ region, it is clear that $H-2$ modifies $\mathrm{H}-\mathrm{Y}$ immunogenicity in some way.

The $I$ region of the $H-2$ complex is known to code for a series of genes which control the immune response to thymus-dependent antigens (McDevitt et al. 1972), MLC reactivity (Bach et al. 1972, Meo et al. 1973), graft-versus host-reactivity (Klein and Park 1973) and skin graft rejection (Klein et al. 1976). Donor-recipient differences in the $I A$ subregion will cause rapid graft rejection while $I C$-subregion differences result in rejection but at a considerably slower rate (Klein et al. 1976). Simpson and co-workers (1973) first reported that the $I r$ gene that controls cytotoxic T-cell response to the $\mathrm{H}-\mathrm{Y}$ antigen maps in the $I A$ subregion, and that which controls female response to male skin maps in the $I B$ subregion. The same group (Hurme et al. 1978) subsequently showed the cytotoxic T-cell response to H$\mathrm{Y}$ in vitro to be low, high or intermediate depending on the $H-2$ haplotype of the responder with control located in the $I A, I C$ and $H-2 D$ or $H-2 K$ subregions. The exact location of the control of the cytotoxic response to $\mathrm{H}-\mathrm{Y}$, therefore, is still indefinite and is probably an interaction of several gene products.

DeMott-Friberg and co-workers (1979) have shown that liver-to-kidney tissue grafts of male liver to female kidneys are also under the control of the $\mathrm{H}-2$ complex. Females of strains carrying the $H-2^{b}$ allele strongly reject these grafts but there is a variation in strength of response depending on the background (non- $H-2$ ) portion of the genome. Different strains which share the $H-2^{s}$ allele also behave differently in the male to female, liver-to-kidney grafting system. SJL females which carry the $H-2^{s}$ allele acutely reject SJL male liver grafts. However, B10.S and A.SW mice fail to exhibit this acute rejection phenomenon. Data reported in this communication relate to the location of control of female response to male liver grafts within the $\mathrm{H}-2$ complex.

Mice of the following strains were bred in our laboratory at the Ann Arbor Veterans Administration Medical Center: B10.A(3R), B10.A(5R), HTI, B10.A(4R), B10.BYR, B10.A(2R), A.TH, B10.A, B10.HTT, B10.S(9R), A.TL, B10.T(6R) and $\mathrm{C} 3 \mathrm{H}$. The strains purchased from the Jackson Laboratory, Bar Harbor, Maine, were: B10.A, B10.A(2R), B10.A(5R), HTG, C3H.HTG, C3H.OH, and B10.DA. The National Cancer Institute provided the following strains: B10.A(5R), B10.A(3R) and B10.HTT. All mice were grafted between the ages of 2 and 6 months. In all strains studied, four mice were killed at 35 days after grafting, and five were killed 70 days after grafting. If histological evaluation did not show good agreement at a particular time, or if the fate of the grafts, i. e., rejection or acceptance, was not obvious for a particular strain, then five grafts were studied at 105 days and/or 140 days after grafting. In some cases, further grafts were studied at 70 and/or 35 days after grafting for confirmatory purposes.

The grafting method is based on the procedure used by Wheeler and co-workers (1966). Our modi operentia have been fully described previously (Schultz et al. 1976). In brief, a small piece of liver taken from an anesthesized donor is placed on the etherized recipient's left kidney which has been prepared by removing a shallow subcapsular slice. The recipient's abdominal incision is sutured and the mouse 
returned to the animal colony. At predetermined times, the recipient mice are killed by cervical dislocation. Grafted kidneys are removed, fixed, trimmed, embedded, sectioned and stained. Numerous sections of each grafted kidney are screened to locate the central area of the graft. Three slides containing sections from the most central areas of the graft are then evaluated.

The criteria for evaluation of graft results and the evaluation methodologies have previously been explained in detail (Schultz et al. 1978). Briefly, catagories "C-1" through "C-6" are increasing in rejection severity with catagories " $\mathrm{C}-5$ " and "C-6" describing early and late stages of complete rejection. Category "P" is a proliferative pattern which is probably a result of immunological activity and " $\mathrm{S}$ " is the designation for a syngeneic-like histological picture.

The results of this series of experiments are summarized in Figure 1. A number of female recombinant mice were grafted with male liver in this series of experiments. Grafted animals were killed 35 and 70 days after grafting. Histological preparations were made and evaluated as described above. Between 3 and 12 animals were evaluated after each time period. In several cases, animals were killed 105 and 140 days after grafting.

The horizontal lines in Figure 1 indicate the range of immunological activity with $\mathrm{P}$ (proliferation) at the low end of the scale and C5-6 (rejection) at the high end of the scale. The vertical marks indicate the mean immunological reactivity for that particular strain at the indicated time after grafting. In strains in which 105 and/or 140 day grafts were evaluated the results did not differ significantly from those found in 70-day grafts. Two sets of previously published skin-graft data have been included in Figure 1 for purposes of comparison.

The first eight strains listed are $H-2^{b}$ recombinants. Virtually all female mice which carry the $H-2^{b}$ haplotype reject male liver by 35 days after grafting (DeMottFriberg et al. 1979). Strains B10.A(3R) and B10.A(5R) differ in the $I J$ region of the $\bar{H}-2$ complex only. Both of these strains show a wide range of graft results at all the times observed. In both strains, many grafts are surviving even at 140 days after grafting, but considerable immunologic activity exists. These strains carry the $K$ end of the $H-2^{b}$ haplotype. B10.A(3R) is $I J^{b}$ while $\mathrm{B} 10 . \mathrm{A}(5 \mathrm{R})$ is $I J^{k}$.

The next six strains listed in Figure 1 carry a number of subregions of the $H-2^{b}$ allele in their $H-2$ recombinant haplotype. Only one strain (HTI) is $H-2^{b}$ at the $K$ end of the $H-2$ complex (subregions $K$ through $I C$ ). At 35 days one of four HTI grafts is C-4 while the other grafts of that strain show only minimal activity. At 70 days the most immunological activity observed in this strain is at the C-2 level.

B10.A(4R) carries the $H-2^{b}$ allele in all but the $K$ and $I A$ subregions. The highest level of activity shown by animals of this strain over a 105 day period is $\mathrm{C}-2$.

The other four strains in the group are $H-2^{b}$ only in the $D$ subregion. None of these strains shows a level of immunological activity greater than C-2.

The next six strains listed in Figure 1 carry the $H-2^{s}$ allele in some position of their $H-2$ genome. A variety of reactivity is shown in these strains ranging from proliferation to $\mathrm{C}-3$ but with most animals categorized as syngeneic-like, C-1 or proliferative. These $\mathrm{H}-2^{s}$-bearing animals have background genomes which are either B10 or A. The first five carry the $H-2^{s}$ allele in their $K$ subregion. None of these five are $H-2^{s}$ in their $D$ subregion. B10.DA is $H-2^{s}$ in its $D$ subregion only, the rest of its $H-2$ complex being $H-2^{q}$. Females of this strain show reactivities between $\mathrm{C}-1$ and 


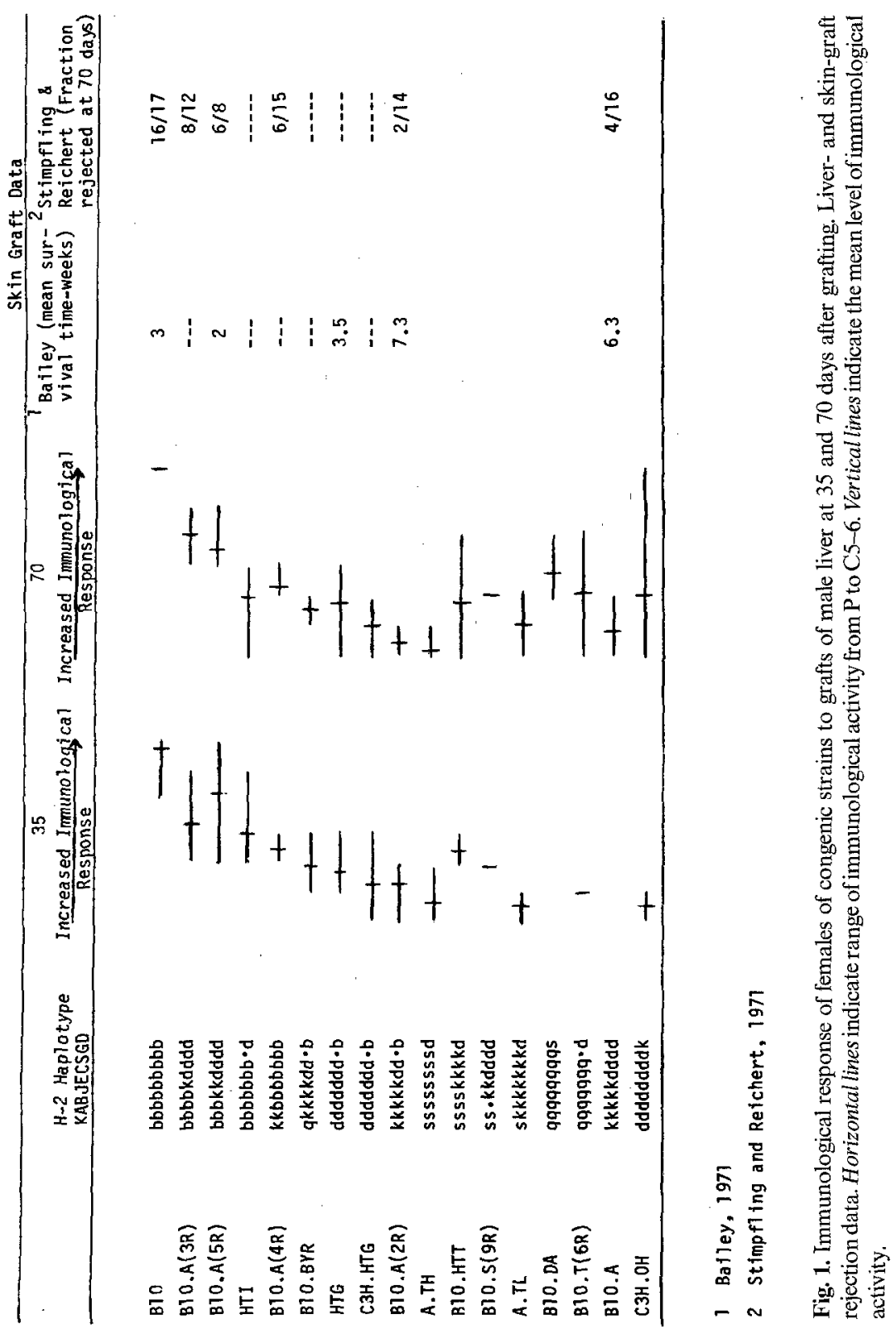


C-3, both 70 and 105 days after grafting. B10.T(6R) which is also $H-2^{q}$ except in its $D$ subregion, which is $H-2^{d}$, shows a somewhat different pattern of reactivity with more proliferative and syngeneic-like grafts than are observed in B10.DA.

The final two strains studied which are $d$ - $k$ recombinants show a.wide range of reactivity from $35-140$ days with a large number of proliferative and mildly reactive grafts.

The study described above provides information pertaining to the location of control of the reaction of female mice to male tissue grafts. Only those strains which carry the $H-2^{b}$ allele in all the subregions of their $H-2$ complex continue to show rejection of liver grafts by 35 days after grafting. When any subregion or subregions of the complex are other than $H-2^{b}$, grafts survive, although they are often under immunologic attack as long as 210 days after grafting. This is true even if all the subregions are $H-2^{b}$ save the $D$ subregion as in strain HTI.

All strains which are $H-2 K^{b}$ show moderately strong immunologic activity at all times observed after grafting [B10.A(3R), B10.A(5R), HTI]. However, if a strain is $H-2^{b}$ only to the right of the $I B$ region, immunologic reactivity is minimal and often less than that found in strains which carry no $H-2^{b}$ subregions. It is clear, therefore, that major control of immunologic activity against the $\mathrm{H}-\mathrm{Y}$ antigen in the liver-graft system is located to the left of the $I A$ region, at least for the $H-2^{b}$ recombinants. The positive effect of the non- $H-2$ portion of the genome in this system is demonstrated by the relatively low immunological activity seen in HTI compared with the activity shown in B10.A (3R) and B10.A (5R). All of these strains are $H-2^{b}$ at the $K$ end of the complex, but HTI's background genome is unique.

The effect of background in this system is also demonstrated by comparing C3H.HTG and HTG. The background genome of HTG combines C57BL/10 and $\mathrm{Balb} / \mathrm{c}$ while the background of $\mathrm{C} 3 \mathrm{H} . \mathrm{HTG}$ is $\mathrm{C} 3 \mathrm{H}$. When $\mathrm{C} 3 \mathrm{H}$.HTG females are grafted with the male liver, few inflammatory cells are found in the graft and the grafts are nearly all proliferative or syngeneic-like. $\mathrm{C} 3 \mathrm{H}$ male to female grafts show similar proliferation. However, HTG animals have many more inflammatory cells infiltrating the graft as well as more hepatic cell destruction.

It has previously been reported that the $H-2 D$ region has considerable control over the fate of male-to-female skin grafts (Gasser and Silvers 1972). This was not found to be true in this system. When strains B10.A(2R) and B10.A are compared, it is clear that their reactivities are very similar. $H-2 D^{b}$ therefore does not confer any stronger reactivity than $H-2 D^{d}$. Strain comparisons of immunological activities are similar for skin grafts (Bailey 1971, Stimpfling and Reichert 1971) and liver grafts (Fig. 1).

Different immunological mechanisms appear to result in proliferation of maleto-female liver grafts in different strains. These mechanistic differences manifest themselves in different ways in the quantity as well as the quality of inflammatory cells in proliferating grafts among recombinant strains. For example, male-tofemale liver-tissue grafts in $\mathrm{C} 3 \mathrm{H} . \mathrm{OH}$ animals contain large numbers of plasma cells and polymorphonuclear leukocytes. B10.T(6R) grafts contain some lymphocytes, but most strains have virtually no inflammatory cells around their proliferating grafts. These differences may be strain-dependent and, therefore, under genetic control. Alternatively, they may be dependent on environmental factors or may result from genetic-environmental interactions. The quality and quantity of cells 
surrounding these grafts and the genetic control of these parameters will be discussed in a future publication.

A comparison of results of male to female liver grafts among recombinants and the parental strains does not facilitate location of control of graft rejection either in the $D$ or $K$ end of the $H-2$ complex. In those recombinant strains for which data on parentals are available (DeMott-Friberg et al. 1979) two strains [B10.A(3R) and $\mathrm{B} 10 . \mathrm{A}(5 \mathrm{R})]$, yielded results which lie between those of their respective parental strains. Two strains, B10.A(4R) and B10.A(2R), gave graft results which more nearly matched those of the $K$-end parent and three strains, A.TH, B10.HTT, and $\mathrm{B} 10 . \mathrm{S}(9 \mathrm{R})$, gave results more like those of the $D$-end parent. Immunologic response to grafts did not segregate with either $Q a$ or Tla.

We must conclude from our data that genetic control over the rejection of male grafts by females of the same strain is a complex interaction of multiple genetic factors. No single gene or even two or three genes may be implicated. The only absolutely clear conclusion is that the $H-2^{b}$ allele located in the $K$ end of the $H-2$ complex controls a stronger immunological response to these tissue grafts than any other allele or subregion. Non- $H-2$ immune-response genes do have an influence on male liver-graft rejection in some recombinant strains but females which are $H-2^{k}$ or $H-2^{d}$ do not reject grafts of their male partner's liver.

Acknowledgements. This study was supported by the Medical Research Service of the Veterans Administration. We wish to thank Mary Bouwsma for excellent technical assistance and Jean Coulter for typing the manuscript.

\section{References}

Bach, F. H., Widmer, M. B., Segall, M., Bach, M. L., and Klein, J.: Genetic and immunological complexity of major histocompatibility regions. Science 176: 1024-1037, 1972

Bailey, D. W.: Allelic forms of a gene controlling the female immune response to the male antigen in mice. Transplantation 11: 426-428, 1971

Bailey, D. W. and Hoste, J.: A gene governing the female immune response to the male antigen in mice. Transplantation 11: 404-407, 1971

DeMott-Friberg, R., Beals, T. F., and Schultz, J. S.: $\mathrm{H}-2$ and background influences on tissue grafts across the H-Y barrier. Immunogenetics 9: 369-381, 1979

Gasser, D. L. and Silvers, W. K.: Genetic control of the immune response in mice. III. An association between H-2 type and reaction to H-Y. J. Immunol. 106: 875-876, 1971

Gasser, D. L. and Silvers, W. K.: Genetics and immunology of sex-linked antigens. Adv. Immunol. 15: $215-247,1972$

Hurme, M., Hetherington, C. M., Chandler, P. R., and Simpson, E.: Cytotoxic T-cell responses to H-Y: mapping of the Ir genes. J. Exp. Med. 147: 758-767, 1978

Klein, J., Geib, R., Chiang, C., and Hauptfeld, V.: Histocompatibility antigen controlled by the I region of the murine H-2 complex. Mapping of the H-2A and H-2C loci. J. Exp. Med. 143: 1439-1452, 1976

Klein, J. and Park, J. M.: Graft-versus host reaction across different regions of the $\mathrm{H}-2$ complex of the mouse. J. Exp. Med. 137: 1213-1255, 1973

McDevitt, H. O., Deak, B. D., Shreffler, D. C., Klein, J., Stimpfling, J. H., and Snell, G. D.: Genetic control of the immune response. Mapping of the Ir-1 locus. J. Exp. Med. 135: 1259-1278, 1972

Meo, T., Vives, J., Miggiano, V., and Shreffler, D.: A major role for the Ir-1 region of the mouse H-2 complex in the mixed leukocyte reaction. Transplant. Proc. 5: 377-381, 1973

Schultz, J. S., Beals, T. F., and Petraitis, F. P.: Tissue graft rejection in mice. I. Contributions of $\mathrm{H}-2$ and non- $-\mathrm{H}-2$ genetic barriers. Immunogenetics 3: 85-96, 1976 
Schultz, J. S., Beals, T. F., and DeMott-Friberg, R.: Tissue graft rejection in mice. V. Survival, rejection, or proliferation across minor barriers. Immunogenetics 6: 585-595, 1978

Simpson, E., Hurme, M., Chandler, P., and Hetherington, C.: Ir Gene Control of T cell responses to the male specific antigen H-Y. J. Immunol. 121: 1614, 1973

Stimpfling, J. H. and Reichert, A. E.: Male-specific graft rejection and the H-2 locus. Transplantation 12: 527-531, 1971

von Boehmer, H., Haas, W., and Jerne, N. K.: Major histocompatibility complex-linked immune responsiveness is acquired by thymocytes of low responder mice differentiated in thymus of highresponder mice. Proc. Natl. Acad. Sei. U.S.A. 75: 2439-2441, 1978

Wachtel, S. S., Gasser, D. L., and Silvers, W. K.: Male-specific antigen: Modification of potency by the H-2 locus in mice. Science 181: 862, 1973

Wheeler, H. B., Carson, J. M., and Dammin, G. L.: Transplantation of tissue slices in mice. Ann. N.Y. Acad. Sci. 129: 118-125, 1966

Received March 3, 1980; revised version received August 12, 1980 